\title{
Pedobarographic Analysis Following Ponseti Treatment for Congenital Clubfoot
}

\author{
Marc F. Sinclair MD, Kerstin Bosch PhD, \\ Dieter Rosenbaum PhD, Stephanie Böhm MD
}

Published online: 28 February 2009

(C) The Association of Bone and Joint Surgeons 2009

\begin{abstract}
Current methods of treating congenital clubfeet provide high rates of functional outcomes. Despite the clinical outcomes, radiographic assessment suggests residual equinus deformity of the hindfoot. It is unclear whether these deformities result in abnormal foot-floor pressures and whether they correlate with clinical outcome. We evaluated 28 feet in 20 patients following Ponseti treatment for clubfoot by clinical and pedobarographic examination a mean of 33 months after removal of the last cast. The data were compared to age- and weight-matched normal subjects and to the unaffected foot in the unilaterally affected patients. Despite ankle range of motion of $30^{\circ}$ and a physiologic hindfoot valgus alignment in 19 cases, pedobarography suggested differences in maximum force, impulse, contact area, and peak pressure compared to normal subjects. Compared to the
\end{abstract}

Each author certifies that he or she has no commercial associations (eg, consultancies, stock ownership, equity interest, patent/licensing arrangements, etc) that might pose a conflict of interest in connection with the submitted article.

Each author certifies that his or her institution has waived or does not require approval for the human protocol for this investigation and that all investigations were conducted in conformity with ethical principles of research.

M. F. Sinclair $(\bowtie)$

Orthopaedic Department, Medcare Hospital, PO Box 215565, Dubai, UAE

e-mail: mmjvsinclair@hotmail.com;

marc.sinclair@medcarehospital.com

K. Bosch, D. Rosenbaum

Funktionsbereich Bewegungsanalytik, Klinik für Allgemeine

Orthopädie, Universitätsklinikum Münster, Munster, Germany

S. Böhm

UKBB, Kinderorthopädische Abteilung, Basel, Switzerland unaffected foot the only difference was reduced peak pressure over the medial hindfoot and forefoot with increased pressure over the lateral midfoot. Similar to radiographic abnormalities in studies on treated clubfeet with good functional outcome, pedobarographic analyses show differences compared to a control group. The value of pedobarographic analysis for predicting successful treatment of congenital clubfoot is questionable since it does not correlate with the clinical outcome in patients treated with the Ponseti method.

Level of Evidence: Level IV, diagnostic study. See the Guidelines for Authors for a complete description of levels of evidence.

\section{Introduction}

The nonoperative treatment of clubfoot deformity developed by Dr. Ignacio Ponseti has gained increasing acceptance as the treatment modality of choice $[13,15,16]$. Weekly manipulation and casting corrects the deformity at the level of the subtalar joint in most cases. If required, a percutaneous tenotomy of the tendoachilles is performed to improve dorsiflexion. To avoid early recurrences, the application of a foot abduction brace is required for approximately 4 years [21]. Initially the brace is applied full time for 3 months, followed by a 14-hour application at night and during naps for the remaining time. Initial correction is reported in up to $98 \%$ of cases $[19,20]$. Compliance with the brace protocol is crucial to avoid recurrences at an early stage [1, 10, 19, 24]. Excellent longterm function has been documented by Cooper and Dietz [5] and Ippolito et al. [15].

To assess the functional load-bearing aspect of the feet during walking, many researchers have used measurements 
of ground reaction forces with force or pressure platforms. In contrast to measurements with a force platform, plantar pressure measurements provide detailed information about the whole foot contact and loading in various foot regions during the roll over process (eg, the normal transition from heelstrike to foot flat and toe off). Pressure measurements analyze the force across a defined surface whereas force describes the interaction between two bodies. Plantar pressure measurements provide several parameters of interest (eg, peak pressure, contact area, contact time, maximum force, and force time integral) and can be recorded for barefoot or in-shoe patterns. Platforms are mostly used in laboratory settings and are typically embedded in a walkway or the floor. Insoles can be used to measure plantar pressures within a shoe to evaluate the effect of different construction or design features of a shoe [6].

Despite good functional outcomes [5, 19], radiographic studies continue to reveal a number of differences between clubfeet and unaffected feet $[5,16,22]$. As a result, standard radiographs as an outcome measure following clubfoot treatment are not frequently obtained or reported. Since pedobarography is sensitive for detecting changes in foot-floor or foot-shoe pressures and since residual hindfoot deformities might create abnormalities, we reasoned barefoot pressures might better correlate with clinical outcomes than radiographs.

The purpose of our study was to ascertain whether pedobarographic measurements would detect differences of idiopathic clubfeet following successful Ponseti treatment when compared to normal feet. Specifically we compared the following pedobarographic parameters of clubfeet with those of normal subjects and those of the normal foot in patients with unilateral clubfoot: (1) peak pressure, (2) maximum force, (3) force time integral, and (4) total contact area.

\section{Materials and Methods}

We identified a consecutive group of 20 children with congenital clubfoot deformity (28 feet) treated by the method described by Ponseti. All patients were available for clinical examination and dynamic foot pressure measurements. Thirteen of the 20 patients were male. The average age at the time this study was conducted was 36.8 months (range, 29-45 months).

The clinical evaluation included range of motion measurement by handheld goniometer reading for hip, knee, and ankle, thigh-foot angle, and the intermalleolar distance. Softness of the heel pad was assessed following Pirani classification (as described by Flynn et al. [9].) and classified as soft or hard, depending on the amount of soft tissue overlying the heel. Range of motion of the subtalar joint and hindfoot alignment were determined as early indicators for possible recurrences [20]. Results from the clubfoot patient group were compared to a control group of normal subjects. The control group consisted of 20 children matched for age, height, and weight with no foot abnormalities or other motor dysfunction. Foot loading parameters of these children have previously been published by Bosch et al. [3] establishing normative values for the developing child. We also compared the data of the healthy versus the affected foot in children with unilateral deformity.

All pedobarographic measurements were performed on an ST 4 platform (EMED ST4, Novel® GmbH, Munich, Germany). The vertical component of ground reaction forces was measured in a $50-\mathrm{Hz}$ frequency enabled by high focal resolution of four sensors $/ \mathrm{cm}^{2}$. All children were instructed to walk barefoot and at a self-selected speed over the platform. After several test runs, measurements were obtained in a standardized fashion. Although total walking distance was not predefined, care was taken that the starting point was in a sufficient distance to the platform, thus avoiding the first step to be on the measuring platform. Only data obtained from an unaided, fluid gait was considered for analysis. Five measurements were obtained for each foot [14]. Data analysis was supported by Novel software (Novel Medical Professional 12.3.19; Novel $\AA$ GmbH, Munich, Germany) and Microsoft Excel (Redmond, WA). The pressure area of the foot was divided into 10 regions of interest according to standard PRC mask provided by the analysis software. The PRC mask was described by Cavanagh et al. [4] and divided the foot in 10 different foot areas: medial and lateral hindfoot (M1 and M2), medial and lateral midfoot (M3 and M4), medial, central, and lateral forefoot (M5, M6 and M7), hallux (M8), second toe (M9), and lateral toes (M10) (Fig. 1).

The boundaries between the heel and the midfoot and the midfoot and the forefoot were defined by default as $73 \%$ and $45 \%$ of the foot length from the toes to the heel respectively. The lateral and medial determination was defined by a foot axis from the center of the heel to the center of the second toe. The first, second and lateral metatarsals heads were separated by straight lines drawn parallel to the foot axis; these lines divided the forefoot region vertically from the medial to the lateral side into sections of $30 \%, 25 \%$, and $45 \%$ respectively (cp. Manual Version 13, Novel GmbH Munich).

We evaluated four dynamic parameters for each area of interest: (1) peak pressure in $\mathrm{kPa}(\mathrm{PP})$ (force divided by area), (2) maximum force in $\mathrm{N}$ (MF), (3) force time integral in Ns (FTI) (integral of force with respect to time), and (4) contact area in $\mathrm{cm}^{2}$ (CA). MF was normalized to 


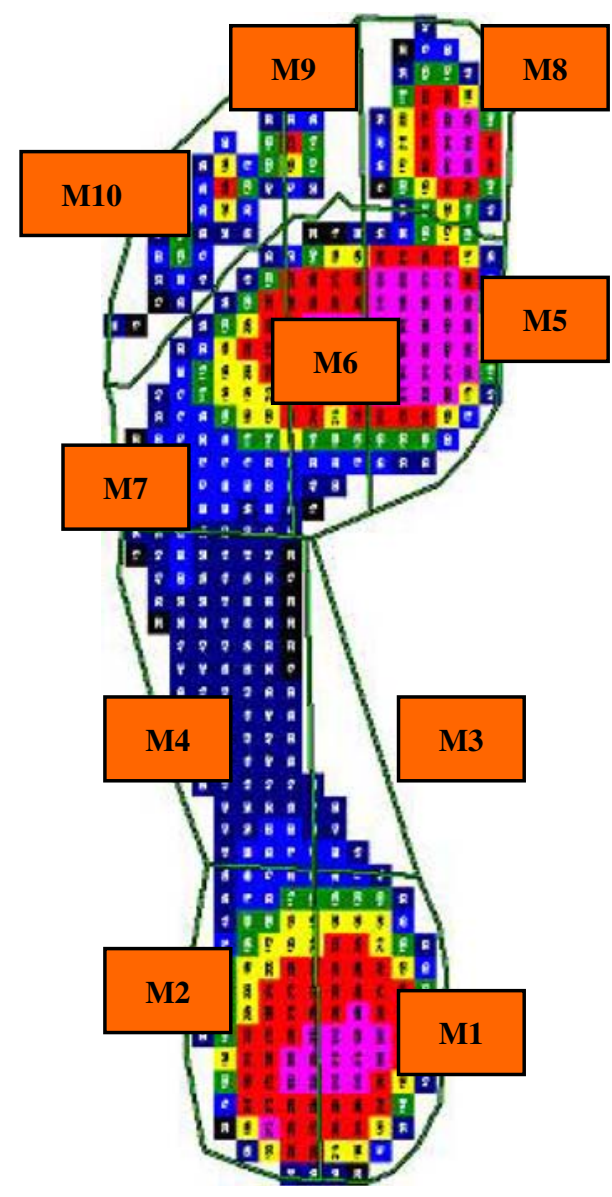

Fig. 1 The PRC mask divides the plantar surface of the foot into 10 predefined areas of interest, creating two separate areas for the medial and lateral hindfoot (M1, M2) and midfoot (M3, M4). The forefoot area and the toe area are each subdivided into a medial, central, and lateral section (M5 to M7 and M8 to M10).

bodyweight (\%BW); CA was normalized to total foot area (\%CA), and FTI was normalized to the force time integral of the total foot (\%FTI).

For analysis we calculated the mean of five foot trials. To obtain five representative trials we sometimes needed to obtain as many as $20-50$ trials. We terminated the trials when five successful trials for each foot were obtained. Twenty trials were typical and did not appear to cause any fatigue in the children. With very few difficult cases we obtained up to 50 trials and then it was more a challenge to keep the children motivated rather than to prevent fatigue; we obtained the trials by "distracting" the child with toys or rewards or games. Trials with incomplete foot prints or double contacts on the platform were omitted. The extracted parameters were not normally distributed as tested by the Kolmogorov-Smirnov one sample test and plotting histograms. Differences in peak pressure, maximum force, force time integral, and contact area between the affected and unaffected foot of the clubfoot group were determined using the nonparametric dependent Wilcoxon signed rank test. Differences between the clubfoot group and the healthy controls were determined using the nonparametric independent Mann-Whitney U test.

\section{Results}

The clinical examination of all clubfeet in the affected group resulted in an average dorsiflexion of the ankle of $30^{\circ}$ and a plantar flexion of $50^{\circ}$. The subtalar joint presented with full range of motion consisting of $40^{\circ}$ of subtalar external rotation. The thigh-foot angle measured an average of $20^{\circ}$. The hindfoot presented in a valgus alignment in 19 patients, and one patient revealed a hindfoot varus. All patients were able to walk on their toes and heels.

Mean peak pressures were reduced $(\mathrm{p}<0.005)$ in all hindfoot and forefoot areas (M1, 2, 5, 6, 7, 8, 9, 10) in the clubfoot group compared with those of the control group (Table 1) (Fig. 4). No difference was observed between the two groups in the midfoot areas (M3, M4) (Fig. 2). When comparing the clubfeet in unilateral affected individuals with the unaffected side, decreased values for mean peak pressure were noted for the medial hindfoot (M1), medial forefoot (M5), and the toe areas (M8, M9, and M10). A relative increase in peak pressure was noted for the lateral midfoot area (M4) $(\mathrm{p}<0.005)$ (Table 2).

Mean total maximum force (MFto) in the clubfoot group was decreased $(\mathrm{p}=0.0026)$ compared to that in the control group. Area-specific analysis resulted in reductions $(\mathrm{p}<0.0001)$ of MF in the hindfoot (M1, M2) and the forefoot (M5, M6). No differences were seen in areas M7, M9, and M10. MF was increased in the midfoot areas M3 $(\mathrm{p}=0.0006)$ and $\mathrm{M} 4(\mathrm{p}<0.0001)$ (Fig. 3 and Fig. 4). No differences were noted in MFto when comparing unilateral clubfeet to the unaffected side $(\mathrm{p}=0.6379)$.

The mean relative impulse as force time integral (FTI) was reduced in areas M1 $(\mathrm{p}=0.0002), \mathrm{M} 5$, and M6 $(\mathrm{p}=0.0001)$ in the clubfoot group. The same group had increased FTI in both the medial $(\mathrm{M} 3, \mathrm{p}<0.0001)$ and lateral (M4, p < 0.0001) aspect of the midfoot (Fig. 5). No differences appeared when comparing with the unaffected foot $(\mathrm{p}=0.530)$.

Mean total contact area (CA) was similar for clubfoot group and control group (CA-to, $p=0.219$ ). We observed decreased mean CA in M1 and M2 (hindfoot) and M5, M6, M8, and M9, representing the more central and medial aspect of the forefoot. Increased $\mathrm{CA}$ was noted in the midfoot M3 $(p=0.0008)$ and M4 $(p<0.0001)$. Total contact area measurement in the affected and not affected feet in unilateral cases were similar $(\mathrm{p}=0.888)$. 


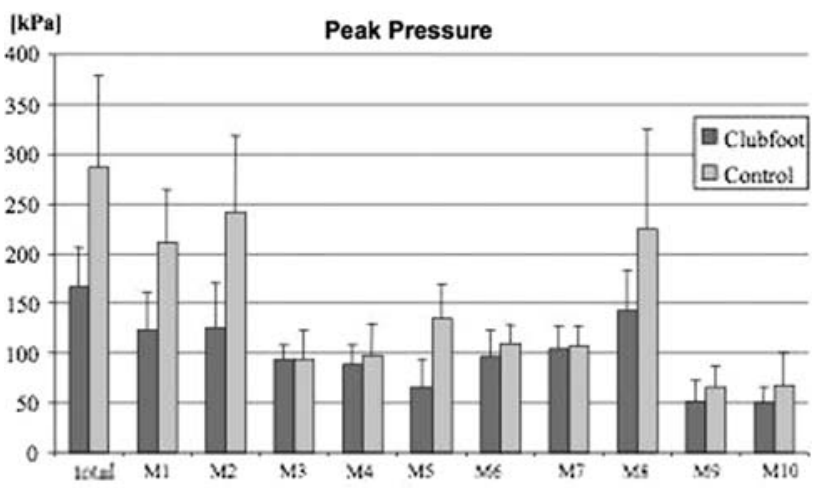

Fig. 2 Decreased peak pressure $(\mathrm{kPa})$ is measured in the clubfoot group in all hindfoot (M1, M2) and forefoot areas (M8 - M10). No differences are noted in the midfoot area (M3, M4).

\section{Discussion}

The treatment protocols for congenital clubfoot deformity have changed substantially in many institutions over the past 10 years. Feet treated by the Ponseti method have improved clinical outcomes when compared to surgically treated feet [13, 15]. Although excellent in functional outcome, radiographic studies suggest residual deformity such as a decreased talocalcaneal angle [5]. The functional load-bearing aspect of the feet during walking can be measured by pressure platforms. Pedobarography analyzes the force across a defined surface by providing several parameters of interest such as peak pressure, contact area, contact time, maximum force, and force time integral and is an objective dynamic test for evaluation of foot function. We wondered whether the typical sorts of residual hindfoot deformities might be reflected in functional load-bearings pattern of successfully treated clubfeet using the Ponseti method. The variables analyzed include a clinical examination and four pedobarographic parameters from peak pressure, total maximum force, force time integral to total contact area.

One limitation to our study is the lack of radiographs. Radler et al. [23] reported that clubfeet treated with the Ponseti method showing dorsiflexion on clinical examination can present with an increased tibiocalcaneal angle suggesting rocker-bottom deformity prior to tenotomy. Even after tenotomy, radiographs suggested a tibiocalcaneal angle below the normal range in 12 of 87 clubfeet. We were not able to include radiographs in our study as parental consent was not given in the majority of cases. Second is the young age of our patients increasing the number of trials necessary in order to obtain five valid trials. Our center, however, has extensive experience in pedobarographic measurements [3] and other similar studies in even younger children have been published by similar experienced groups in the past [7]. Further, 


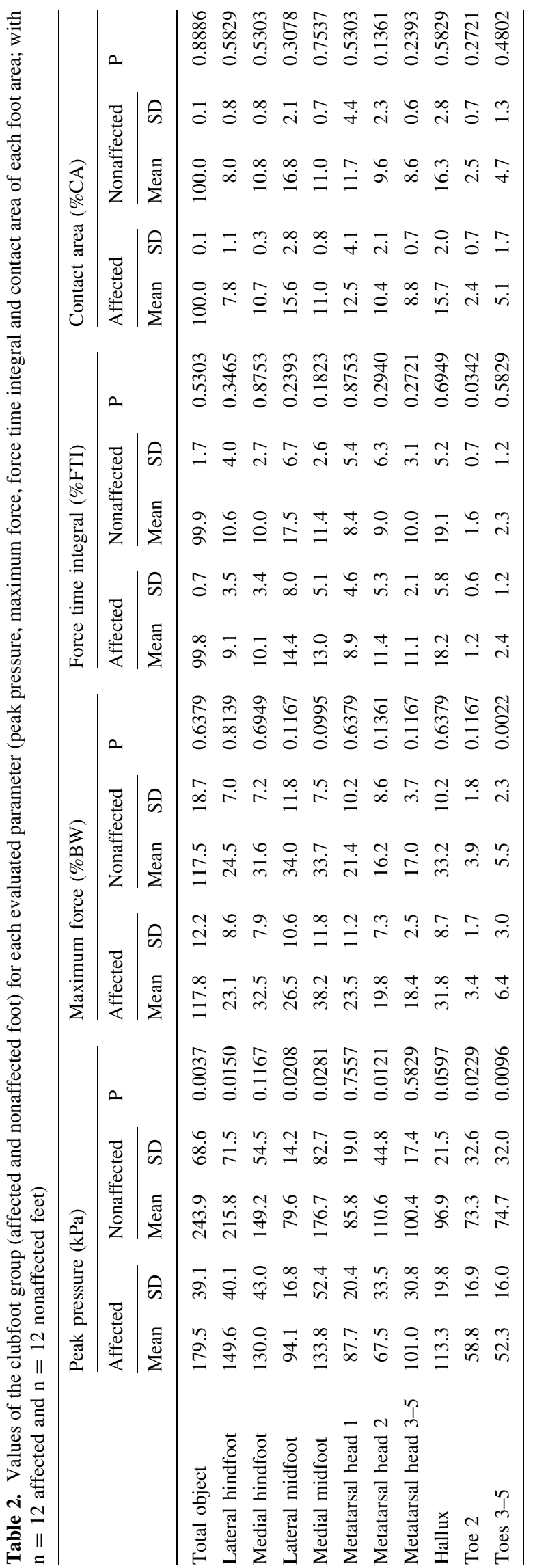

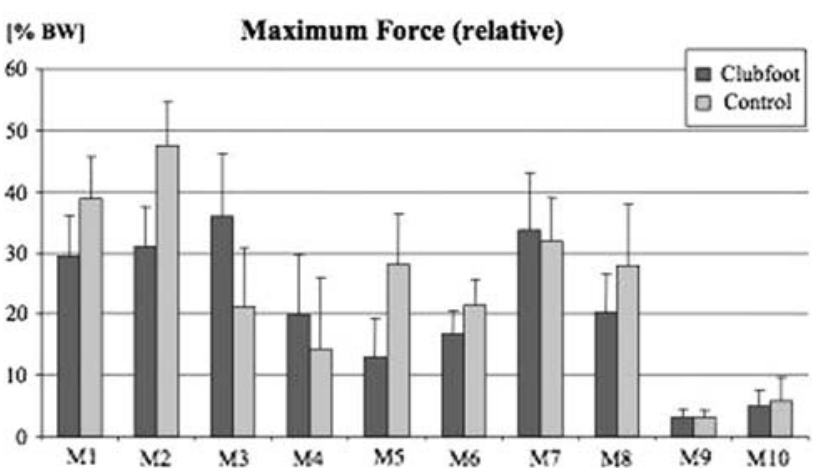

Fig. 3 Maximum force (\% BW) for the clubfoot group was decreased in all hindfoot areas (M1, M2) and medial and central forefoot areas (M5, M6). Increased values were noted for the midfoot areas M3 and M4 within the clubfoot group.

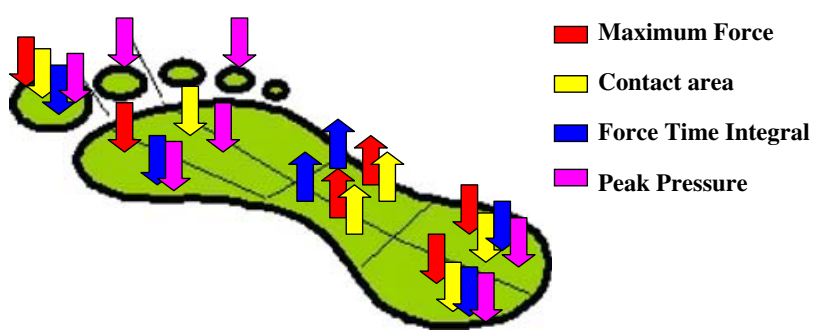

Fig. 4 Compared data from clubfoot patients with those of the control group suggest decreased values for maximum force (MF), contact area (CA), force time integral (FTI), and peak pressure (PP) in both hindfoot areas as well as the medial toe and forefoot areas. FTI, $\mathrm{PP}$, and CA show increased values for the medial and lateral midfoot area.

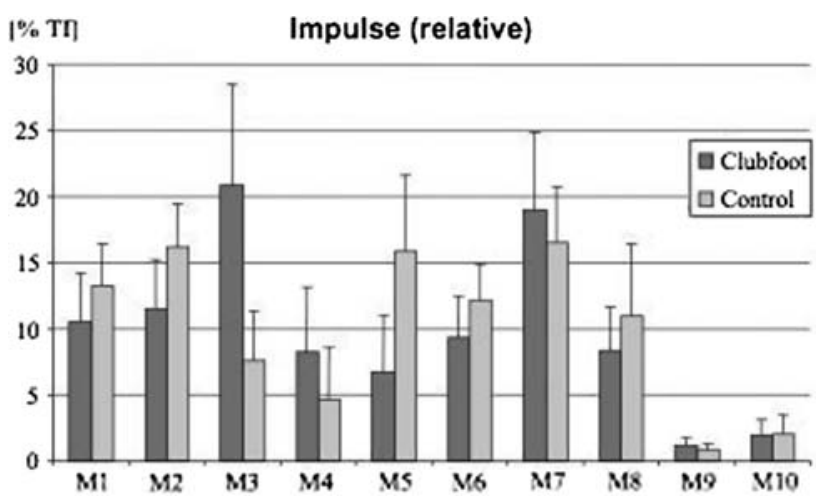

Fig. 5 Impulse measured as force time integral (\%TI) for the clubfoot group shows an increase for both midfoot areas (M3, M4) with moderately reduced values for the hindfoot (M1, M2) and the forefoot (M5, M6 and M8).

targeting the pressure plate has not in the past influenced the ground reaction force parameters when using our gait protocol (free walking at self-selected speed and without covering the platform; previously described by Wearing et al. [25].) in combination with experienced personnel. 
Fig. 6 Examples for peak pressure and resulting center of pressure (COP) path in the clubfoot and control group show widening of the hind and midfoot areas with reduced peak pressure over the hindfoot in the Ponseti group. No lateralization of the COP path is seen.
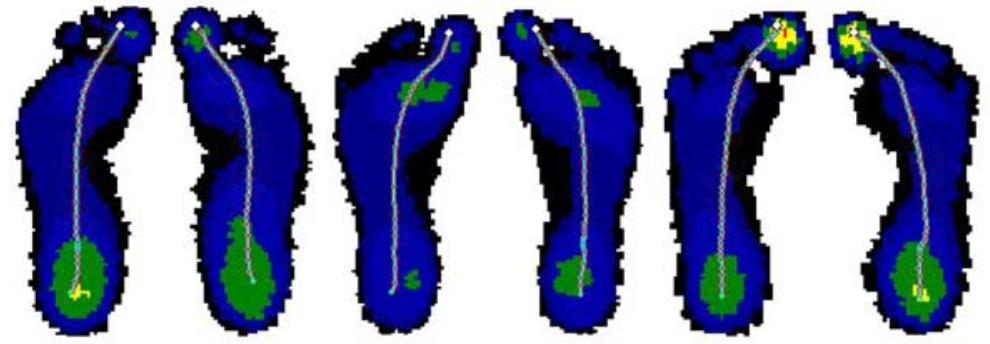

Ponseti group
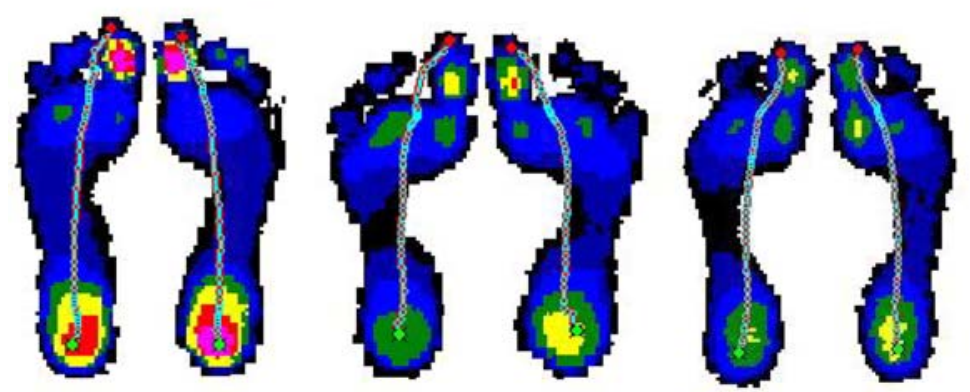

Finally, the small number of patients in our clubfoot group may reduce the generalizability of our observations. Rather than stating statistical significance, we included all $p$ values for the reader's consideration (Tables 1,2). However, all children in this group were treated by one physician following the same treatment protocol resulting in a highly homogeneous group.

In 1995, Cooper and Dietz [5] examined 45 adults with 71 clubfeet treated by the Ponseti method. In their followup study, patients were evaluated clinically, radiographically, and by pedobarographic and electrogoniometric measurements. Clinically, the examined feet showed mildly reduced dorsiflexion of the ankle and on radiological evaluation presented with residual cavus deformity. The pedobarographic evaluation revealed increased pressure over the midfoot area similar to our present study. The change in pressure pattern was attributed to a reduced dorsiflexion causing a shift from the hindfoot to the midfoot area. Although we were able to measure increased peak pressure in the midfoot area, our patients did not have any limitation of their ankle motion with an average of $30^{\circ}$. All clubfeet however revealed a soft heel pad according to the Pirani classification [9] which in our opinion could be the leading cause for reduced peak pressure of the hindfoot area in the examined clubfeet [5]. The amount of fatty tissue under the sole has been seen as a possible cause for reduced values of hindfoot peak pressure when comparing the plantar pressure distribution in children with that of adults $[12,18]$. An increased fat pad of the heel is frequently seen in children after Ponseti treatment, providing a possible explanation for reduced peak pressure of the hindfoot (Fig. 6). According to Morag and Cavanagh, a decreased calcaneal inclination angle will decrease peak pressure under the heel and increase pressure under the midfoot [18]. Their model suggests an increased calcaneal inclination of one standard deviation will reduce peak pressure of the midfoot by $20.2 \mathrm{kPa}$. Radiographic analyses by Radler et al. [23] report an average lateral talocalcalcaneal angle of $36.4^{\circ}$ with 12 of 87 clubfeet showing angles below the reported normal range. An increase in midfoot pressure, however, does not necessarily lead to the conclusion of a rocker-bottom foot in feet with normal ankle dorsiflexion. Cooper and Dietz reported increased midfoot pressures in clubfeet with a radiographically measurable midfoot cavus [5]. The average age at followup was 34 years, however, and allows no direct comparison to our younger population.

Hee et al. similarly concluded normal-looking clubfeet with good function are in fact not normal by gait analysis or static pedobarography [11] with increased mid and forefoot pressure in their surgically treated clubfoot group. Similarly our patients with good range of motion also showed elevated maximum force, contact area, and impulse of the midfoot with reduction of the same parameters in the forefoot and hindfoot areas in the pedobarographic analysis. We also observed reduced peak pressures of the heel and first toe, widening of the hind- and midfoot area. The center of pressure path however showed only minimal changes in comparison to the control group (Fig. 6). Lateralization of the center of pressure path towards the outer border of the foot has previously been described for surgically treated clubfeet with persistent varus of the hindfoot and midfoot adductus [26]. However, one study comparing Ponseti-treated clubfeet with the agematched control group and in unilateral cases with the contralateral unaffected side [17] reported no clear tendency toward lateralization. A mild lateral transfer of weight bearing was also reported by Cooper and Dietz [5] 
with, however, no effect on the overall functional result. They suggest a certain tolerance towards a shift of the center of pressure path.

Aronson et al. [2] reported no differences in morphometric analysis, strength, radiographic structure, and movement when comparing the unaffected foot of children with unilateral clubfoot with a matched control group. In contrast to these results, Favre et al. [8] suggested that even the unaffected foot in unilaterally affected children exhibited differences in pressure and force distribution when compared to normal feet. According to these authors, a smaller ankle plantarflexion moment in the clubfoot might be compensated by the hip and knee joint of the same limb resulting in an asymmetrical gait pattern affecting pedobarographic parameters of the contralateral side. The differences might also be attributed to a central nervous adaptation of the gait pattern in an attempt to compensate for any existing asymmetries. These asymmetries have been observed not only in the kinematics of the foot itself but also further proximal, such as in a lower gastrocnemius activity on the unaffected side in patients with one clubfoot [17]. It is therefore questionable if the contralateral foot in unilaterally affected individuals can be used as a reliable control group. Our results are consistent with these sorts of asymmetries, showing differences only in peak pressure measurement between the two feet. Similar to Favre et al. [8], we found the unaffected foot had increased peak pressure for the areas M1, M8, and M10, representing the area of the medial hindfoot, hallux, and third to fifth toe, with decreased pressure in the lateral midfoot area. There may also be mechanical influence of the soft tissue around the heel pad on hindfoot peak pressure [18], resulting in decreased pressure values with increasing tissue thickness. Frequently clubfeet treated with the Ponseti method initially have a softer heel. This persisting softness reduces over the first two to three years and results in a heel with normal soft tissue distribution (personal communication, Dr. Ignacio Ponseti). Differences in peak pressure between the affected and unaffected foot in unilaterally affected individuals might relate to basic asymmetries in the gait [8] or to tissue differences, whereas the remaining measurements of maximal force, contact area, and impulse measurement are not influenced by this condition.

We report a pedobarographic analysis of 20 children with congenital clubfoot deformity at an average of 33 months after Ponseti treatment. Despite excellent dorsiflexion and full ankle range of motion, the clubfoot group showed differences when compared to an age-matched control group. When using the unaffected foot as a control in patients with unilateral clubfoot, we found an increase only in peak pressure measurement for the lateral midfoot and decreased values for the medial hindfoot, hallux, and lateral toe area. Future studies need to correlate radiographic results with pedobarographic measurements to identify any clinical implication of the differences obtained by pedobarographic analysis in asymptomatic clubfeet treated with the Ponseti method and understand their causes if clinically important.

\section{References}

1. Abdelgawad AA, Lehman WB, van Bosse HJ, Scher DM, Sala DA. Treatment of idiopathic clubfoot using the Ponseti method: minimum 2-year follow-up. J Pediatr Orthop B. 2007;16:98-105.

2. Aronson J, Puskarich CL. Deformity and disability from treated clubfoot. J Pediatr Orthop. 1990;10:109-119.

3. Bosch K, Gerss J, Rosenbaum D. Preliminary normative values for foot loading parameters of the developing child. Gait Posture. 2007;26:238-247.

4. Cavanagh PR, Rodgers MM, Iiboshi A. Pressure distribution under symptom-free feet during barefoot standing. Foot Ankle. 1987;7:262-276.

5. Cooper DM, Dietz FR. Treatment of idiopathic clubfoot. A thirtyyear follow-up note. J Bone Joint Surg Am. 1995;77:1477-1489.

6. Dixon SJ. Use of pressure insoles to compare in-shoe loading for modern running shoes. Ergonomics. 2008;51:1503-1514.

7. El-Hawary R, Karol LA, Jeans KA, Richards BS. Gait analysis of children treated for clubfoot with physical therapy or the Ponseti cast technique. J Bone Joint Surg Am. 2008;90:1508-1516.

8. Favre P, Exner GU, Drerup B, Schmid D, Wetz HH, Jacob HA. The contralateral foot in children with unilateral clubfoot: a study of pressures and forces involved in gait. $J$ Pediatr Orthop. 2007;27:54-59.

9. Flynn JM, Donohoe M, Mackenzie WG. An independent assessment of two clubfoot-classification systems. J Pediatr Orthop. 1998;18:323-327.

10. Haft GF, Walker CG, Crawford HA. Early clubfoot recurrence after use of the Ponseti method in a New Zealand population. J Bone Joint Surg Am. 2007;89:487-493.

11. Hee HT, Lee EH, Lee GS. Gait and pedobarographic patterns of surgically treated clubfeet. J Foot Ankle Surg. 2001;40:287-294.

12. Hennig EM, Staats A, Rosenbaum D. Plantar pressure distribution patterns of young school children in comparison to adults. Foot Ankle Int. 1994;15:35-40.

13. Herzenberg JE, Radler C, Bor N. Ponseti versus traditional methods of casting for idiopathic clubfoot. $J$ Pediatr Orthop. 2002;22:517-521.

14. Hughes J, Pratt L, Linge K, Clark P, Klenerman L. Reliability of pressure measurements: the EMED F system. Clin Biomech. 1991;6:14-18.

15. Ippolito E, Farsetti P, Caterini R, Tudisco C. Long-term comparative results in patients with congenital clubfoot treated with two different protocols. J Bone Joint Surg Am. 2003;85:1286-1294.

16. Laaveg SJ, Ponseti IV. Long-term results of treatment of congenital club foot. J Bone Joint Surg Am. 1980;62:23-31.

17. Maton B, Wicart P. Centrally adaptations in unilateral idiopathic clubfoot children following conservative treatment. $J$ Electromyogr Kinesiol. 2005;15:72-82.

18. Morag E, Cavanagh PR. Structural and functional predictors of regional peak pressures under the foot during walking. $J$ Biomech. 1999;32:359-370.

19. Morcuende JA. Congenital idiopathic clubfoot: prevention of late deformity and disability by conservative treatment with the Ponseti technique. Pediatr Ann. 2006;35:128-30, 132-136. 
20. Morcuende JA, Abbasi D, Dolan LA, Ponseti IV. Results of an accelerated Ponseti protocol for clubfoot. J Pediatr Orthop. 2005;25:623-626.

21. Morcuende JA, Dolan LA, Dietz FR, Ponseti IV. Radical reduction in the rate of extensive corrective surgery for clubfoot using the Ponseti method. Pediatrics. 2004;113:376-380.

22. Ponseti IV, El-Khoury GY, Ippolito E, Weinstein SL. A radiographic study of skeletal deformities in treated clubfeet. Clin Orthop Relat Res. 1981;30-42.

23. Radler C, Manner HM, Suda R, Burghardt R, Herzenberg JE, Ganger R, Grill F. Radiographic evaluation of idiopathic clubfeet undergoing Ponseti treatment. J Bone Joint Surg Am. 2007;89: $1177-1183$.

24. Thacker MM, Scher DM, Sala DA, van Bosse HJ, Feldman DS, Lehman WB. Use of the foot abduction orthosis following Ponseti casts: is it essential? J Pediatr Orthop. 2005;25:225-228.

25. Wearing S, Urry S, Smeathers J. The effect of visual targeting on ground reaction force and temporospatial parameters of gait. Clin Biomech. 2000;15:583-591.

26. Widhe T, Berggren L. Gait analysis and dynamic foot pressure in the assessment of treated clubfoot. Foot Ankle Int. 1994;15: $186-190$. 